\title{
Numerical Study of Natural Convection Heat Transfer of a Square Eccentric Body Buried in a Porous Media
}

\author{
Ramiz Ibraheem saeed \\ Asst.lecturer \\ Ra'ed Ahmed Ali \\ Asst.lecturer \\ University of Mosul- College of Engineering- Mech. Engineering Department.
}

\begin{abstract}
Natural convection in a saturated porous medium bounded by two horizontal eccentric ducts is studied numerically by solving the governing Darcy-Boussinsq equations using finite differences on very fine grid. This paper reports the effect of the position of a cooled square body with respect to the centre of the square cavity vertically and horizontally. The numerical results of heat transfer are presented for modified Rayleigh number $\mathrm{Ra}^{*}$ (based on width of cavity) in the range (50 to 400) and for different size of the body with respect to the width of the cavity $(0.25 \times 0.25,0.3 \times 0.3,0.5 \times 0.5)$ and six positions of the body respect to the center of cavity. The results are represented by streamlines and isotherms to show the behavior of the flow and temperatures distribution. Also some graphics had been obtained which represent the relationship between Nusselt and Rayleigh numbers with eccentricity. (Key words: natural convection, porous medium, cavity)

Key Words : Porous medium, Thermal nonequilibrium, Heat transfer, Natural convection.

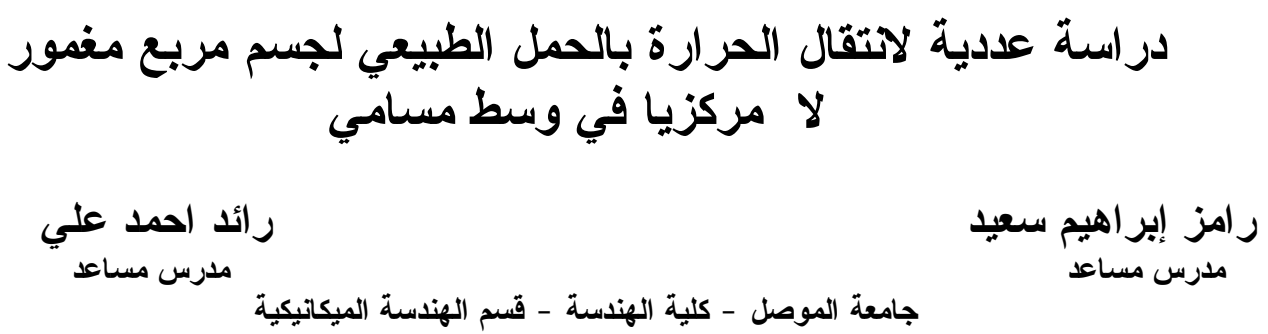

\section{الخلاصة}

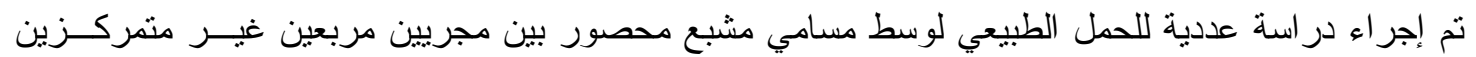

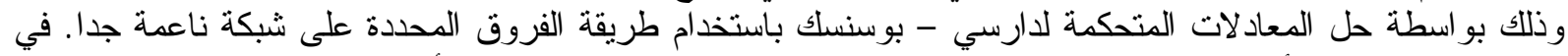

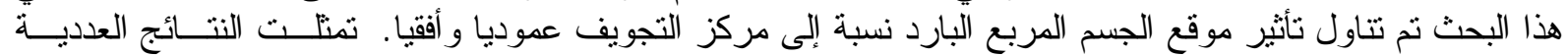

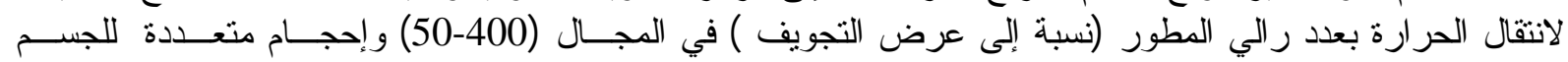

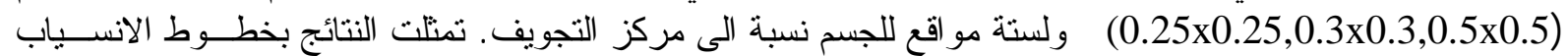

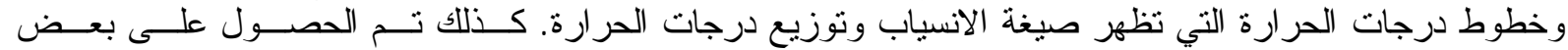
الرسومات البيانية التي منلت آلعلاقة بين عدد نسلت و عدد ر التي مع التلا تمركزية.
\end{abstract}




\section{Nomenclature}

$\mathrm{C}_{\mathrm{p}} \quad$ Specific heat at constant pressure, $(\mathrm{J} / \mathrm{kg} . \mathrm{K})$

$\mathrm{Da} \quad$ Darcy number, $\mathrm{K} / \mathrm{L}^{2}$

$\mathrm{e}_{\mathrm{x}} \quad$ Horizontal eccentric distance between the center of cavity and center of body

$\mathrm{e}_{\mathrm{y}} \quad$ Vertical eccentric distance between the center of cavity and center of body

F Arbitrary function

G Acceleration due to gravity, $\left(\mathrm{m} / \mathrm{s}^{2}\right)$

$\mathrm{K} \quad$ Permeability of porous medium, $\left(\mathrm{m}^{2}\right)$

$\mathrm{k}_{\mathrm{e}} \quad$ effective thermal conductivity of porous medium, (W/m.K)

$\mathrm{L} \quad$ Length of side of porous cavity, (m)

$\mathrm{L}_{\mathrm{b}} \quad$ Length of side of body, (m)

$\mathrm{Nu} \quad$ Nusselt number, $\mathrm{Q}_{\text {conv }} / \mathrm{Q}_{\text {cond. }}$

$P \quad$ Pressure, $N / \mathrm{m}^{2}$

Q Quantity of heat transfer (W)

$\mathrm{Q}_{\text {cond. }} \quad$ Quantity of heat transfer by conduction (W)

Qconv. Quantity of heat transfer by convection (W)

$\mathrm{Ra} \quad$ Rayleigh number, $\rho_{\mathrm{o}} \mathrm{g} \beta \mathrm{L}^{3} \Delta \mathrm{T} / \mu \alpha$

$\mathrm{Ra}^{*} \quad$ Modified Rayleigh number, Da . Ra

$\mathrm{T}$ Dimensional temperature, $(\mathrm{K})$

$\Delta \mathrm{T} \quad$ Temperature difference, $\mathrm{T}_{\mathrm{h}}-\mathrm{T}_{\mathrm{c},}(\mathrm{K})$

$\mathrm{u}, \mathrm{v}$ Darcian velocity components, $\mathrm{m} / \mathrm{s}$

$\mathrm{x}, \mathrm{y} \quad$ Cartesian coordinates

\section{Greek symbols}

$\alpha_{\mathrm{e}} \quad$ Effective thermal diffusivity, $\left(\mathrm{m}^{2} / \mathrm{s}\right)$

$\beta \quad$ Coefficient of thermal expansion of fluid

$\theta \quad$ Dimensionless temperature, $\left(\mathrm{T}-\mathrm{T}_{\mathrm{c}}\right) /\left(\mathrm{T}_{\mathrm{h}}-\mathrm{T}_{\mathrm{c}}\right)$

$\mu \quad$ Dynamic viscosity , $(\mathrm{Kg} / \mathrm{m} . \mathrm{s})$

$\rho \quad$ Density, $\left(\mathrm{Kg} / \mathrm{m}^{3}\right)$

$\Psi \quad$ Stream function, $\left(\mathrm{m}^{2} / \mathrm{s}\right)$

\section{Subscripts}

c Cold

e Effective

$h \quad$ Hot wall

o Reference condition (for the media)

\section{Superscript}

A Dimensionless parameter

Mean 


\section{Saeed: Numerical Study of Natural Convection Heat Transfer of a Square Eccentric}

\section{Introduction}

The analysis of natural convection heat transfer in a porous medium an is important subject due to its increasing applications in many industrial and geophysical problems, particularly in oil exploration and in the petroleum chemical and nuclear industries.

The literature review can be classified into two groups: the first one includes the rectangular cavities and the second one includes bodies embedded in porous media.

Chan et al [1] performed a numerical finite difference solution for a rectangular cavity subjected to different temperatures on its vertical sides . They used a modified Darcy's law which includes the viscous term that becomes important at very high permeability ( Brinkman flow ). The results are presented in terms of theoretical streamlines and isotherms. Upon these, the relative increases in heat transfer rate, corresponding to natural convection, are obtained as functions of three-dimensionless parameters, i.e. the Darcy number, the Rayleigh number, and aspect ratio.

Burns et al [2] examined analytically and numerically convection in a vertical slot filled with porous insulation. This work also included wall injection for both free and forced convections and their results for no wall injection were fairly in agreement with the previous works.

Prasad and Kulaki [3] studied convection numerically, using finite difference method, natural convection in a rectangular porous cavity .The effect of aspect ratio is summarized by a family of curves, for aspect ratio from 0.05 to 100 . It is found that the phenomena of multicellular flow at aspect ratio less than one.

Oothnizen [4] studied two-dimensional flow over a horizontal hot plate in a saturated porous medium mounted near impervious adiabatic horizontal surface and subjected to horizontal forced flow, where Darcy's model is adopted. This problem was numerically investigated by using finite element method. The heat transfer rate from the plate is influenced both by the dimensionless depth of the plate below the surface and the importance of the buoyancy forces.

Barbosa Mota et al [5] studied convection numerically using finite difference to solve the Darcy-Boussinesq equation in 2D for an eccentric horizontal annulus. A mesh containing 1500 grid points to capture the secondary counter-rotating cell in the top of porous layer . For moderate Rayleigh numbers lowering the inner cylinder center leads to a reduction of the overall Nusselt number, the net gain is of the order of $10 \%$.

Al-Ani [6] studied convection numerically using finite differences method, natural convection heat transfer from isothermal rectangular body embedded centerlly in a saturated porous medium. The isothermal body was kept at constant hot temperature and the porous medium has an impermeable rectangular boundaries. Two types of boundary conditions had been used for cavity walls. Different correlations have been made to show the dependence of Nusselt number on modified Rayleigh number at different isothermal body sizes and at different boundary conditions.

Saeed [7] In his investigation, study of two dimensional natural convection heat transfer from isotherms horizontal cylinder embedded in saturated porous medium bounded by squire cavity, carried out numerically. The cylinder is kept at constant hot temperature, while the cavity takes two boundary conditions. The first boundary condition is when all walls of the cavity at constant cold temperature and the second one is when horizontal walls insulated and the vertical walls at constant cold temperature. 
Our main objective here is focusing on eccentricity of the isothermal cold square body embedded in saturated porous medium confined in square cavity and it influences on the heat transfer and Nusselt number.

\section{Mathematical Formulation}

\section{Assumptions :}

1.The porous media is assumed to be rigid and the fluid incompressible with density changes occurring only as result of changes in the temperature according to :

$$
\rho=\rho_{\mathrm{o}}\left[1-\beta\left(\mathrm{T}-\mathrm{T}_{\mathrm{O}}\right)\right]
$$

Where $\rho_{\circ}$ is the fluid density, T the temperature, $\beta$ is the coefficient of thermal expansion. The effects of fluid density changes are accounted for in the buoyancy term in the equations of motion and are neglected elsewhere .

2.All other fluid and solid properties are assumed constant .

3. Fluid and matrix are in thermal equilibrium and isotropic .

4. The porous medium is homogeneous.

5. The flow assumed two dimensional, steady state .

6. The viscous dispersion assumed is neglected and no heat generation .

7. The Darcy's law is assumed valid .

The considered problem is an isothermal eccentric square body with sides $\left(\mathrm{L}_{b}\right)$ embedded in a porous medium bounded by a square cavity. The vertical eccentric distance is $\left(\mathrm{e}_{\mathrm{y}}\right)$ and horizontal eccentric distance is $\left(\mathrm{e}_{\mathrm{x}}\right)$ as shown in figure (1) .

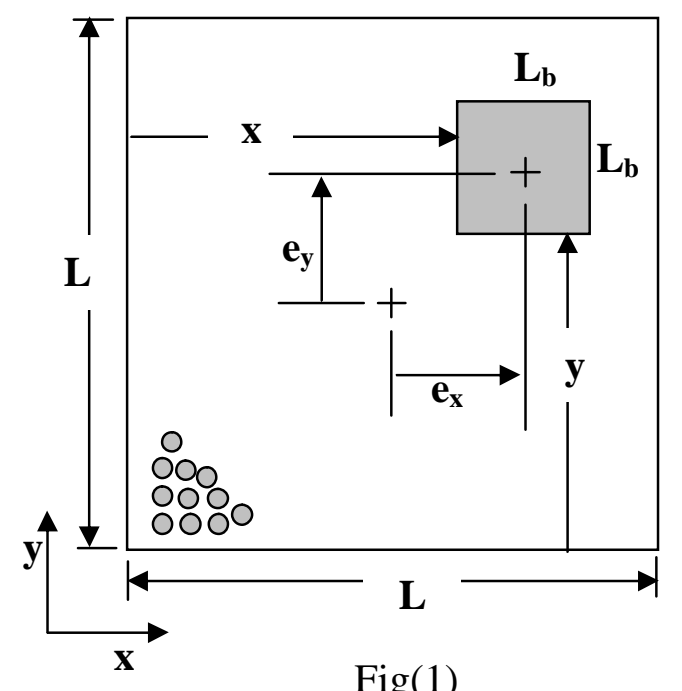

The body is maintained at constant uniform cold temperature and walls of cavity maintained at constant cold temperature. The governing equations that describe the heat transfer process for steady natural convection with Darcy flow and Boussinesq approximation with ( continuity, momentum and energy ) equations are as follows:

$$
\begin{aligned}
& \frac{\partial u}{\partial x}+\frac{\partial v}{\partial y}=0 \\
& \frac{\partial u}{\partial y}-\frac{\partial v}{\partial x}=-\frac{\rho_{\mathrm{o}} g \beta K}{\mu} \frac{\partial T}{\partial x} \\
& u \frac{\partial T}{\partial x}+v \frac{\partial T}{\partial y}=\alpha_{e}\left[\frac{\partial^{2} T}{\partial x^{2}}+\frac{\partial^{2} T}{\partial y^{2}}\right]
\end{aligned}
$$


Where, $\quad u=-\frac{K}{2} \frac{\partial p}{\partial} \quad \mathrm{v}=-\frac{\mathrm{K}}{2}\left(\frac{\partial \mathrm{p}}{\partial \mathrm{p}}+\rho \mathrm{g}\right) \quad$ (Darcy law) $\ldots(5)$

The following variabltes are defined as hordzdimensional:

$$
\theta=\frac{T-T_{c}}{T_{h}-T_{c}} \quad \hat{x}=\frac{x}{L} \quad \hat{y}=\frac{y}{L}
$$

And from the definition of the stream function as:

$$
u=-\frac{\partial \psi}{\partial y} \quad v=\frac{\partial \psi}{\partial x}
$$

And the non dimensional stream function

$$
\hat{\psi}=\frac{1}{\alpha} \psi
$$

Then, the governing equations reduce to :

$$
\begin{gathered}
\frac{\partial^{2} \hat{\psi}}{\partial \hat{x}^{2}}+\frac{\partial^{2} \hat{\psi}}{\partial \hat{y}^{2}}=R a^{*} \frac{\partial \theta}{\partial \hat{x}} \\
\frac{\partial \hat{\psi}}{\partial \hat{x}} \frac{\partial \theta}{\partial \hat{y}}-\frac{\partial \hat{\psi}}{\partial \hat{y}} \frac{\partial \theta}{\partial \hat{x}}=\frac{\partial^{2} \theta}{\partial \hat{x}^{2}}+\frac{\partial^{2} \theta}{\partial \hat{y}^{2}}
\end{gathered}
$$

Where $\mathrm{Ra}^{*}$ is modified Rayleigh number which equals Rayleigh number multiplied by Darcy number (Da).

$$
R a^{*}=R a \times D a \quad \text { Where } \mathrm{Da}=\mathrm{K} / \mathrm{L}^{2}
$$

Boundary conditions:

Cavity:

Body :

$$
\text { at } \hat{x}=0,1 \text { any } \hat{y} \quad \theta=1, \hat{\psi}=0
$$$$
\text { at } \hat{x}=\hat{x}_{b}, \hat{x}_{b}+\hat{L}_{b} \text { and } \hat{y}_{b} \leq \hat{y} \leq \hat{y}_{b}+L_{b} \quad \theta=0, \hat{\psi}=0
$$$$
\text { at } \hat{y}=\hat{y}_{b}, \hat{y}_{b}+\hat{L}_{b} \text { and } \hat{x}_{b} \leq \hat{x} \leq \hat{x}_{b}+L_{b} \theta=0, \hat{\psi}=0
$$

\section{Numerical method}

The numerical method used in this paper is a "finite differences technique", It's procedure is based on the most common series, Taylor series .Using this series, all partial derivatives in governing partial equations are converted to forms that can be handled by computer.

Considering the region that contains the porous matrix is two-dimensional and covered by a cartesian mesh ( $100 \mathrm{x} 100)$. It is considered for a two-dimensional region covered with a rectangular mesh as is shown in figure (2). 


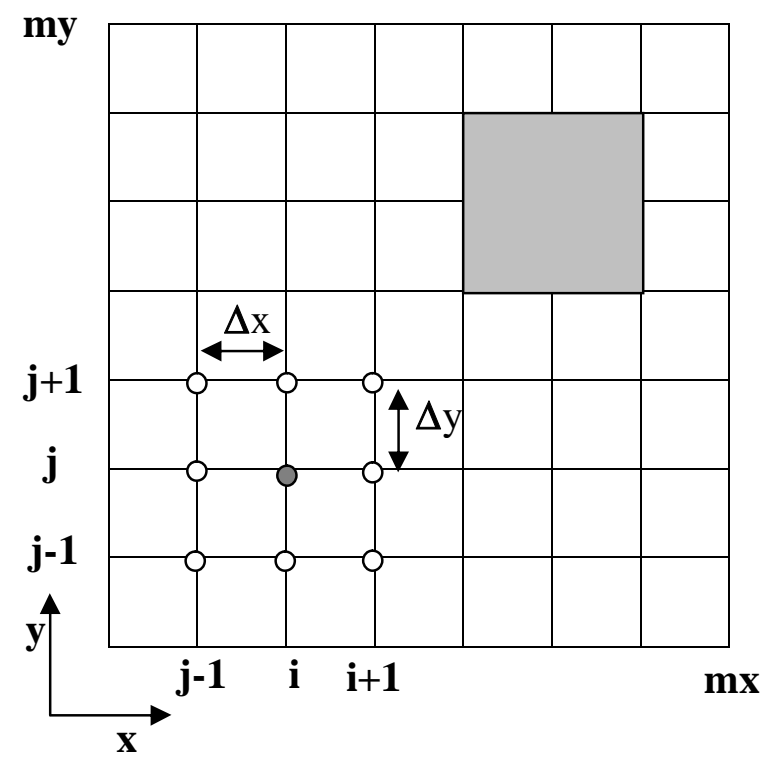

Fig(2) The Cartesian mesh of tow-dimensional domain

The mesh consists of $m x$ vertical and $m y$ horizontal lines positioned at intervals of $\Delta \mathrm{x}$ and $\Delta y$, respectively .Assuming that a function " $f "$ and its derivatives are single-valued, finite and continuous functions of the independent variables $x$ and $y$. Thus, the following finite difference formulas for the first and second order derivatives at grid point $(i, j$ ) from expansions of Taylor series had been obtained :

$$
\begin{aligned}
\frac{\partial f}{\partial x} & =\frac{f_{i+l, j}-f_{i-l, j}}{(1+a) \Delta x}, \quad \frac{\partial f}{\partial y}=\frac{f_{i, j+1}-f_{i, j-1}}{(1+b) \Delta y} \\
\frac{\partial^{2} f}{\partial x^{2}} & =\frac{2}{\Delta x(1+a)}\left(\frac{f_{i+l, j}-f_{i, j}}{a \Delta x}-\frac{f_{i, j}-f_{i-l, j}}{\Delta x}\right) \\
\frac{\partial^{2} f}{\partial y^{2}} & =\frac{2}{\Delta y(1+b)}\left(\frac{f_{i, j+1}-f_{i, j}}{b \Delta y}-\frac{f_{i, j}-f_{i, j-1}}{\Delta y}\right)
\end{aligned}
$$

Therefore, substituting the above three equations in the non-dimensional governing equations obtained previously, to obtain all temperatures and stream functions at each point of region. The quantity of heat transfer from the walls of cavity to the square body can be obtained by using the integration of temperature gradient along the walls of cavity as:

$$
\begin{gathered}
Q=\left.\int_{x=0}^{x=1} \frac{\partial \theta}{\partial \hat{y}}\right|_{a t y=0 \text { or } 1} d \hat{x} \text { (horizontal walls) } \\
Q=\left.\int_{y=0}^{y=1} \frac{\partial \theta}{\partial \hat{x}}\right|_{a t x=0 \text { or } 1} d \hat{y} \text { (vertical walls) }
\end{gathered}
$$

After computing the total quantity heat transfer from the all walls of cavity can be calculate the mean Nusselt number which equals the ratio between quantities of heat transfer by convection to the quantity of heat transfer by pure conduction:

$$
\overline{N u}=\frac{Q_{\text {conv }}}{Q_{\text {cond }}}
$$




\section{Discussion :}

Figure (3) a,b,c shows the effect of the increase in the Rayleigh number on the flow structure and heat transfer rate. It clarifies, during the observation of isotherms lines, that the increase of Rayleigh number brings about increasing of the temperature gradient consequently to leads increasing of heat transfer rate, specifically from the upper part of the body. The appearance of curvatures on isotherms lines with the increasing of Rayleigh number, as reason of the increase in the natural convection activity, results from the influence of bouncy force. This phenomenon occurs in the restricted field between the body and lower surface of the cavity. It can be noticed from the above figure the increase Rayleigh number that gives rise to increasing of fluid velocity. This results from the increase of the bouncy force effect. Also, the great value of streamline function increases with the increase of Rayleigh number causing more activity in natural convection. The appearance of secondary cells which are increasing with the increase of Rayleigh number produces efficient mixing of fluid.
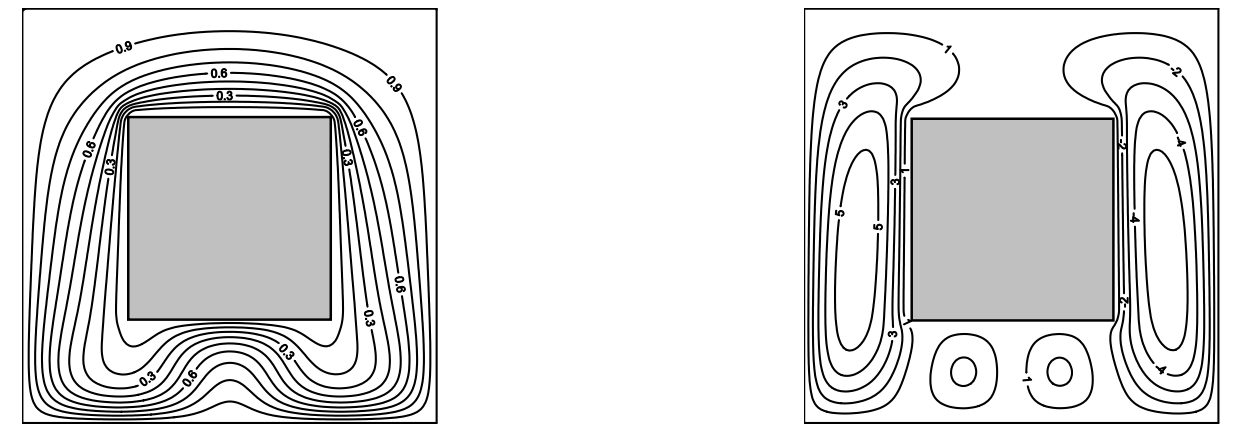

( b )
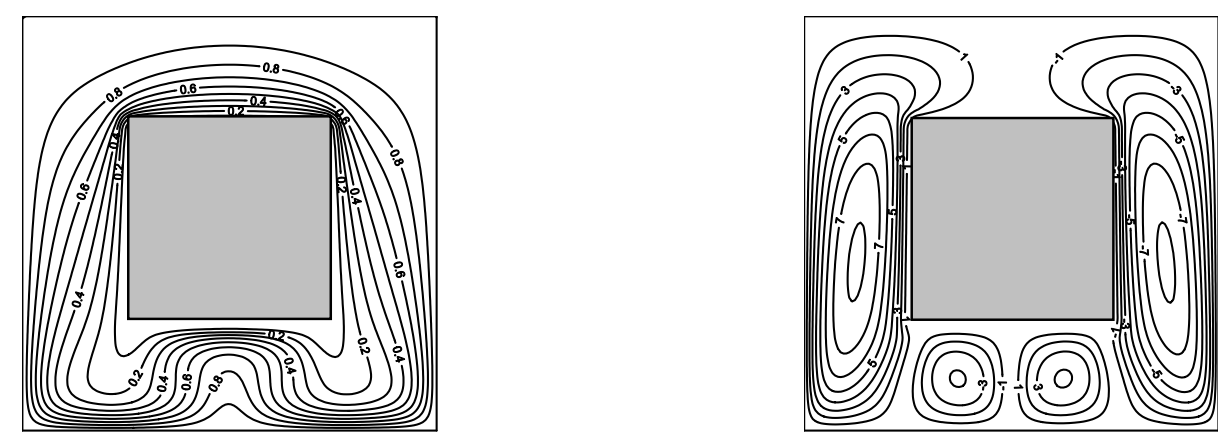

( c )
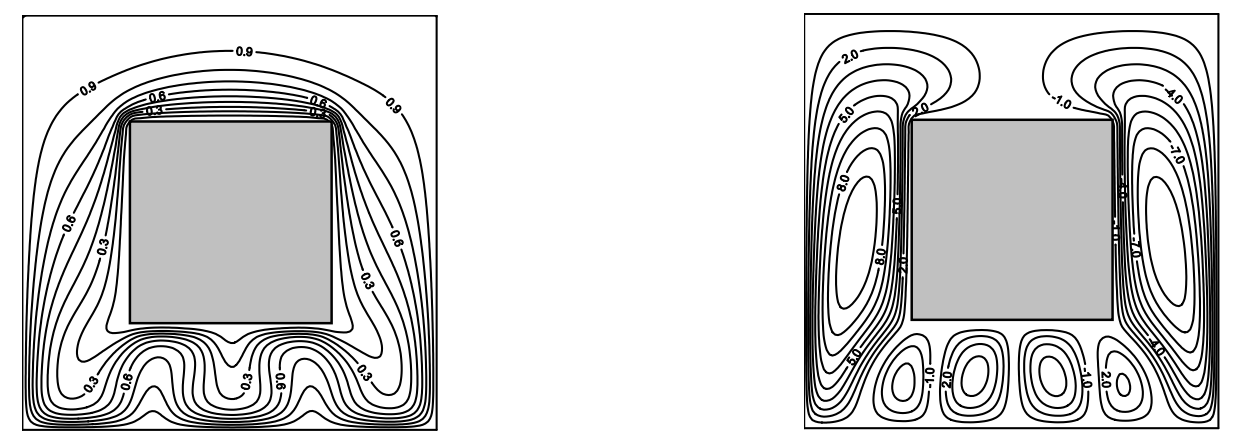

Fig ( 3 ) Isotherms lines \& Stream functions for $\mathrm{e}_{\mathrm{x}}=0, \mathrm{e}_{\mathrm{y}}=0, \mathrm{~L}_{\mathrm{b}}=0.5$

(a) $\mathrm{Ra}=200$, (b) $\mathrm{Ra}=300$, (c) $\mathrm{Ra}=400$ 


\section{Al-Rafidain Engineering $\quad$ Vol.17 $\quad$ No.4 $\quad$ August 2009}

Figure (4) a,b,c clarifies the effect of the body size on streamlines and isotherms lines. It is possible to know the effect of body size increase on heat transfer rate and flow structure through the isotherms lines and streamlines. Whereas the body increment of the body size gives rise to increase of heat transfer rate due to the increase of the temperature gradient. But the influence of body size increase can be observed on streamlines. Also, the increment of body size causes straitening of fluid flowing because of the straitening of the abandoned field in front of the fluid flowing.

( a )
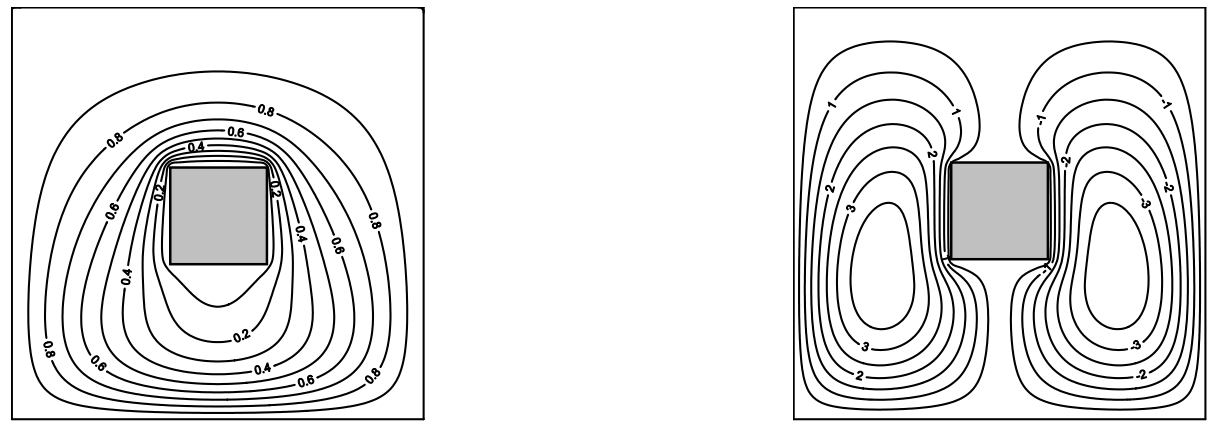

( b )
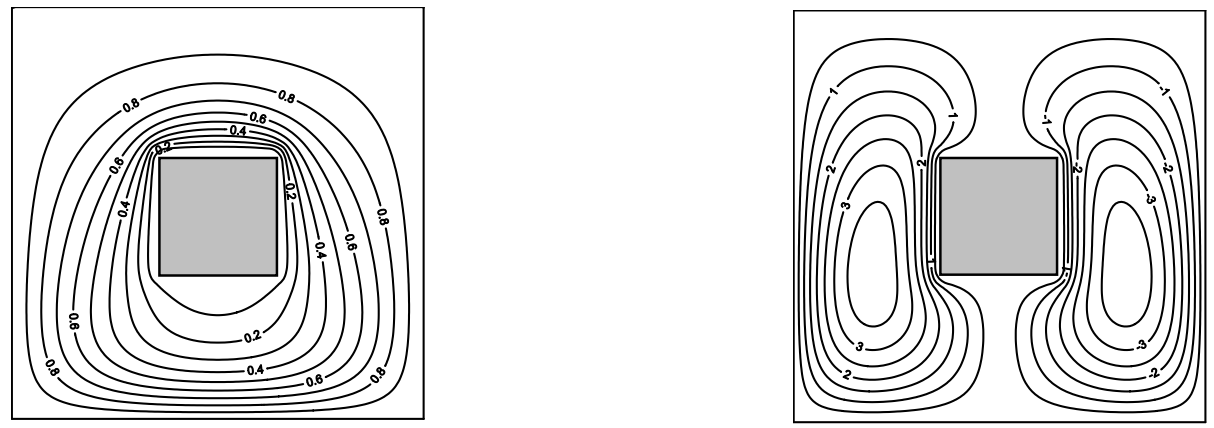

(c)
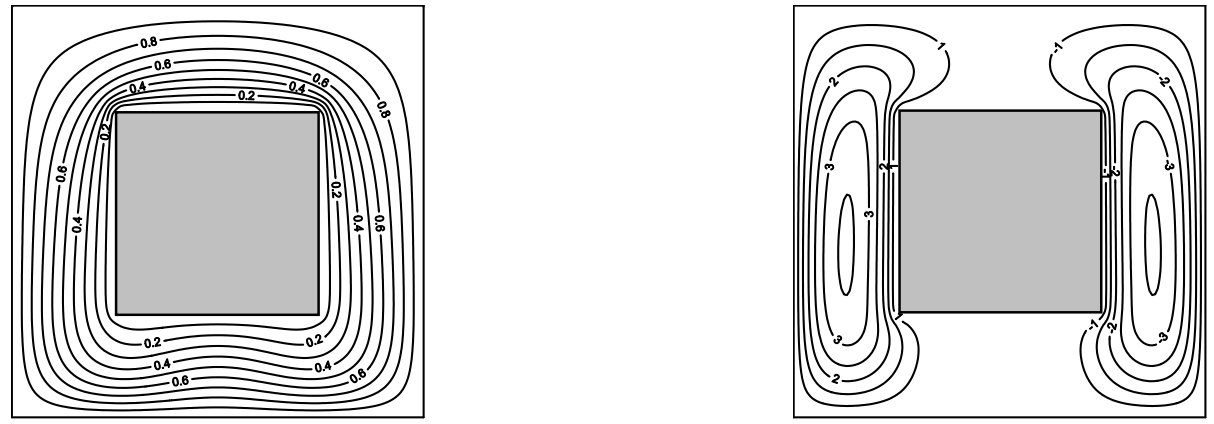

Fig ( 4 ) Isotherms lines \& Stream functions for $\mathrm{e}_{\mathrm{x}}=0, \mathrm{e}_{\mathrm{y}}=0, \mathrm{Ra}=100$ (a) $\mathrm{L}_{b}=0.25$, (b) $\mathrm{L}_{b}=0.3$, (c) $\mathrm{e}_{\mathrm{y}}=\mathrm{L}_{\mathrm{b}}=0.5$ 


\section{Saeed: Numerical Study of Natural Convection Heat Transfer of a Square Eccentric}

Figure (5) a,b,c explains the effect of vertical position of the body on streamlines and isotherms lines. Observing isotherm lines shows that the heat transfer rate increases when the body becomes nearer to the upper surface of the cavity. This results from the increasing in the temperature gradient. Also, noticing the streamlines that the velocity of the fluid flow becomes higher when the body becomes nearer from the upper surface of the cavity, due to the influence of bouncy force which increases more as much as the body becomes nearer to the upper surface of the cavity.

( a )
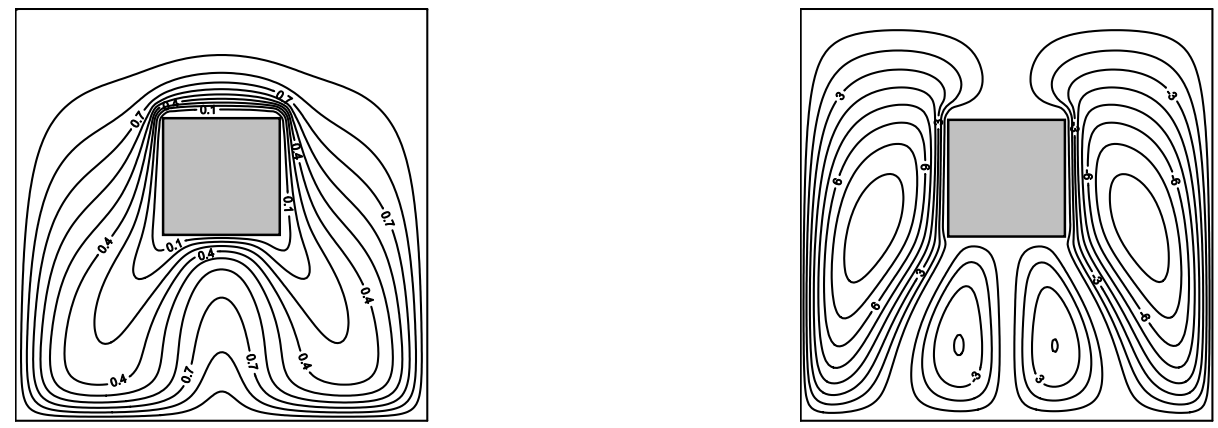

( b )
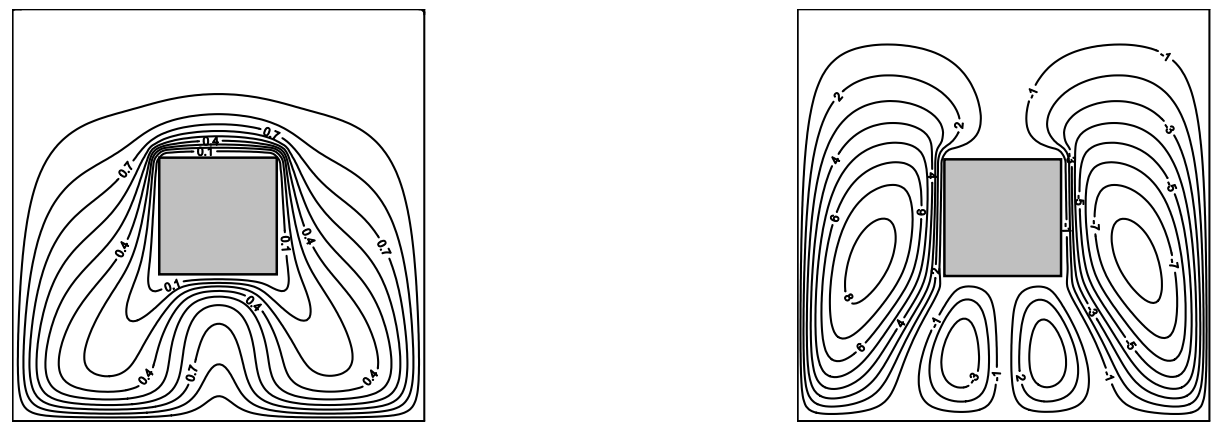

( c )
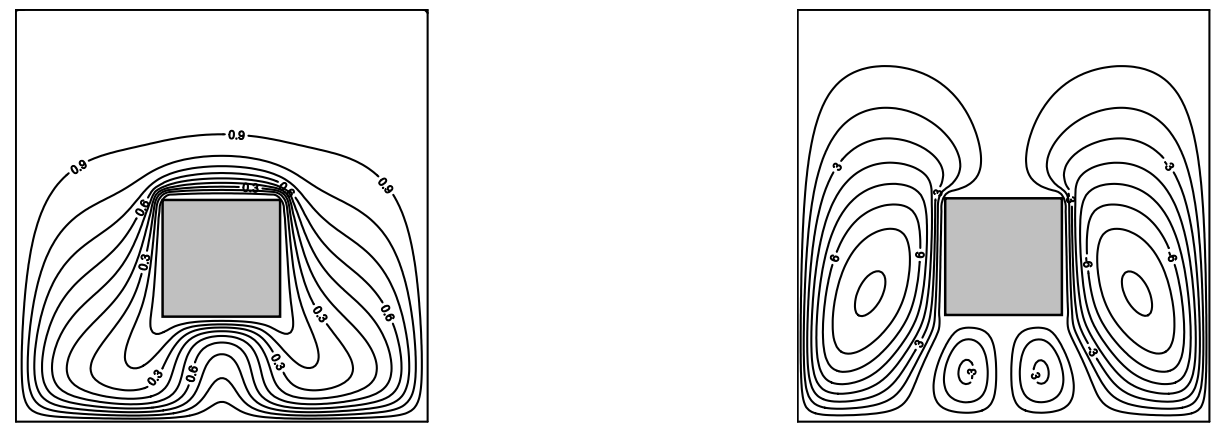

Fig ( 5 ) Isotherms lines \& Stream functions for $\mathrm{L}_{\mathrm{b}}=0.3, \mathrm{e}_{\mathrm{x}}=0, \mathrm{Ra}=300$

(a) $e_{y}=0.1$, , b) $e_{y}=0$, (c) $e_{y}=-0.1$ 


\section{Al-Rafidain Engineering $\quad$ Vol.17 $\quad$ No.4 $\quad$ August 2009}

Figure (6) a,b,c shows the influence of horizontal position of the body on isotherms line and streamlines. The change of the horizontal position of the body has no tangible effect on isotherms lines and this reflects subsequently on the rate of the heat transfer, but the effect of the horizontal position of the body on stream function lines leads to asymmetry of streamlines, due to the variety of the abandoned space in front of fluid flow. In return, this reflects on the great value of stream function $\left(\psi_{\max }\right)$.

( a )
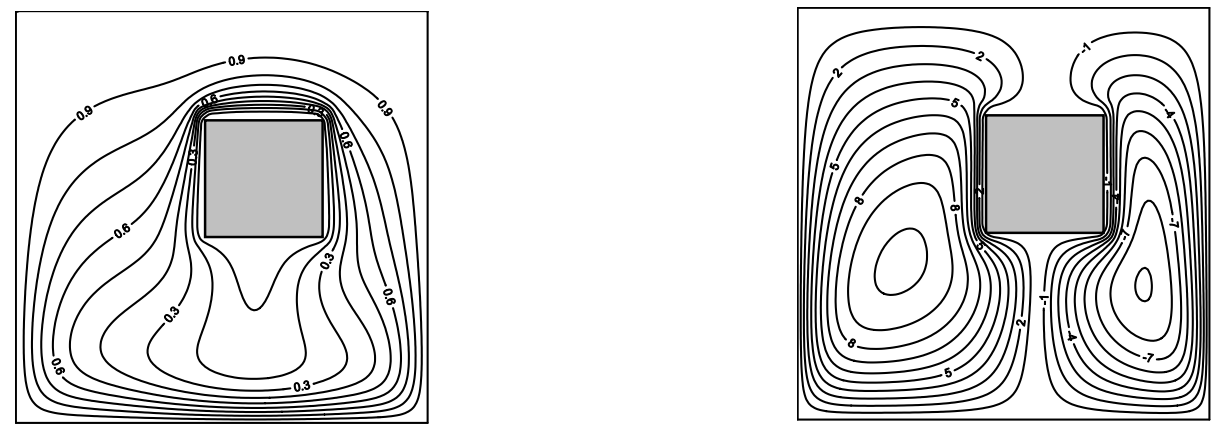

( b )
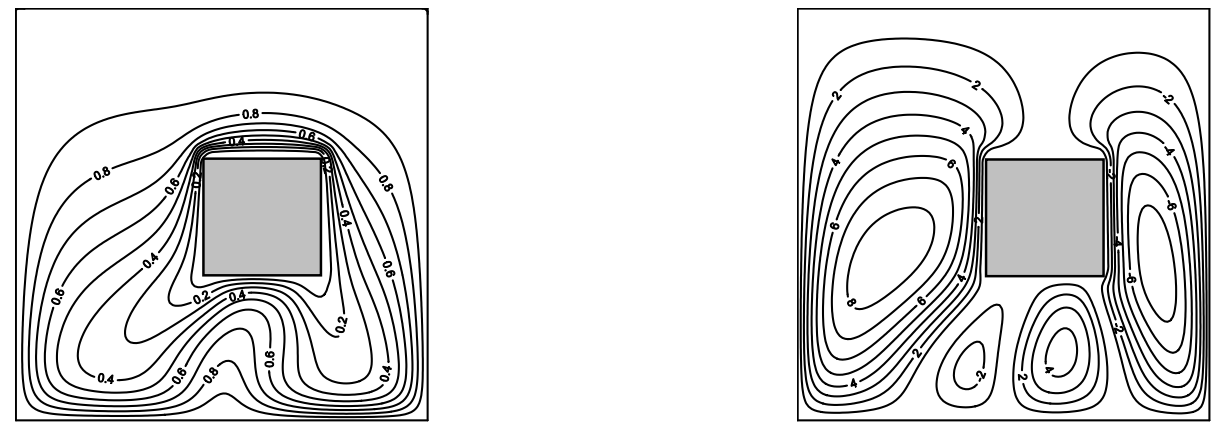

( c )
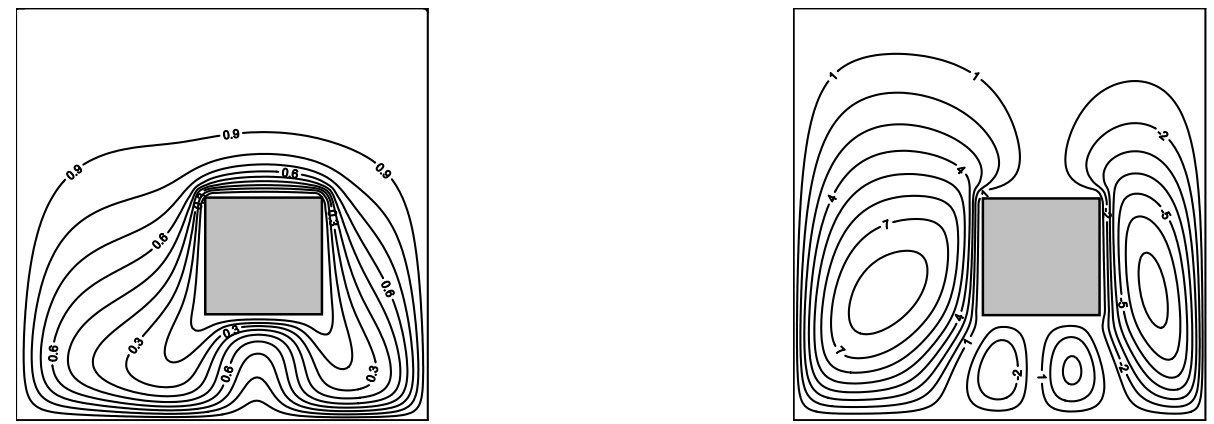

Fig ( 6 ) Isotherms lines \& Stream functions for $\mathrm{L}_{\mathrm{b}}=0.3, \mathrm{e}_{\mathrm{x}}=0.1, \mathrm{Ra}=300$ (a) $\mathrm{e}_{\mathrm{y}}=0.1$, , b) $\mathrm{e}_{\mathrm{y}}=0$, (c) $\mathrm{e}_{\mathrm{y}}=-0.1$ 
Figure (7) a,b,c shows the effect of the Rayleigh number on the Nusselt number. Through the figure can notice that the increase in the Rayleigh number causes the increase in the Nusselt number. For the increase of Rayleigh number resulting in the increase of bouncy force, that causes increasing in heat transfer rate by natural convection and this reflects on Nusselt number. Also, the horizontal position has no tangible effect upon Nusselt number. Moreover, one notice that the Nusselt number value approach to each other. The change of vertical position of the body has an impact on Nusselt number values when the body becomes nearer from the upper surface of the cavity the Nusselt number values becomes greater than other positions.

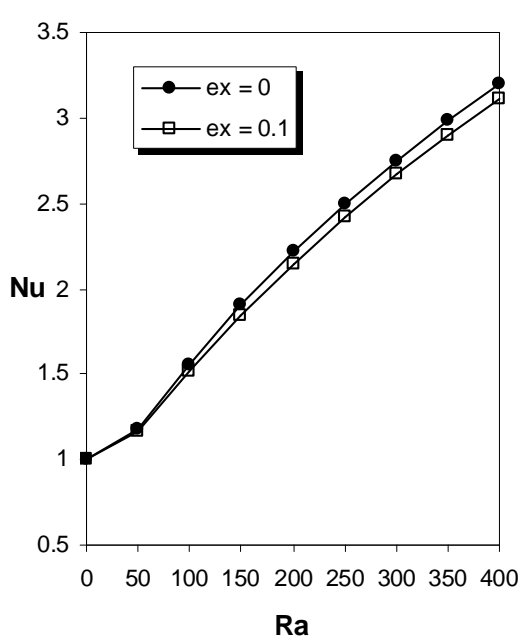

( a )

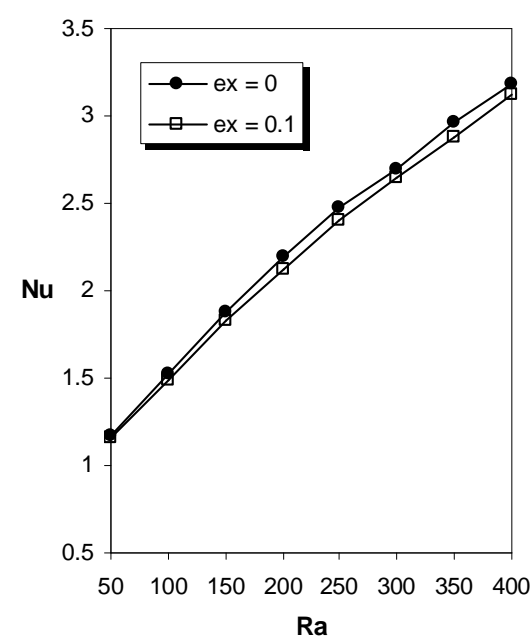

( b )

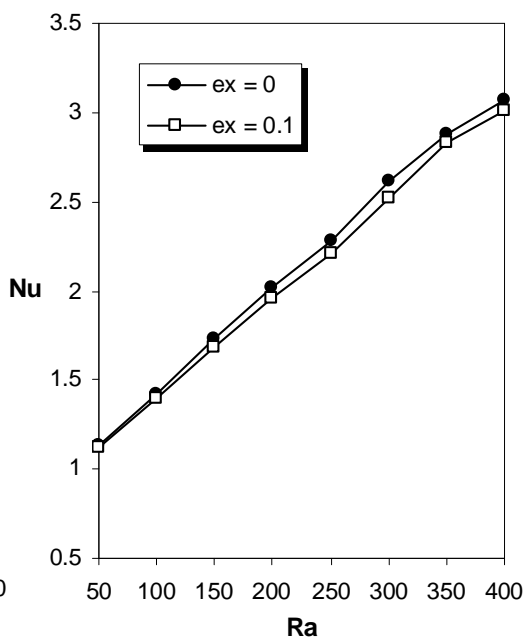

( c )

Fig ( 7 ) Nu versus Ra for $L_{h}=0.25$ (a) $e_{v}=0.1$. (b) $e_{v}=0$. (c) $e_{v}=-0.1$

Figure (8) a,b,c shows the effect of vertical position of the body on the Nusselt number with a change in the Raleigh number and the body size. The values of Nusselt number are at higher degree when the body is closed to the upper surface of the cavity, with variety of Raleigh number values. But when the body size increases and equal to $(0.5 \times 0.5)$, so the value of Nusselt is the greatest when the body is existed in the middle of the cavity due to the striating of abandoned space in front of fluid flowing, so the increase heat transfer rate by conduction greater than the increase in the heat transfer rate by natural convection, so the value of Nusselt number are the greatest when the body is existed in the middle of cavity.

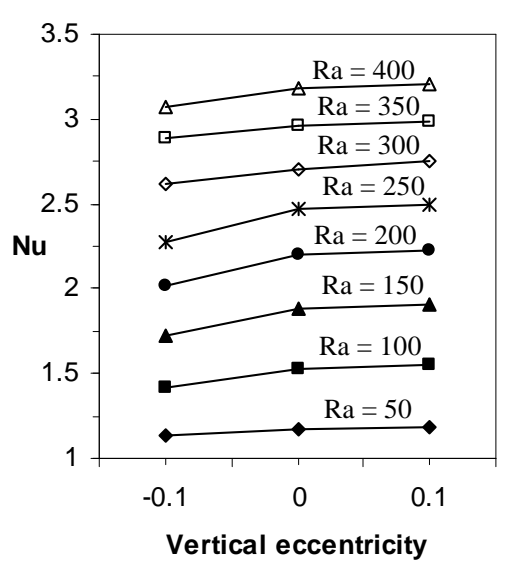

( a )

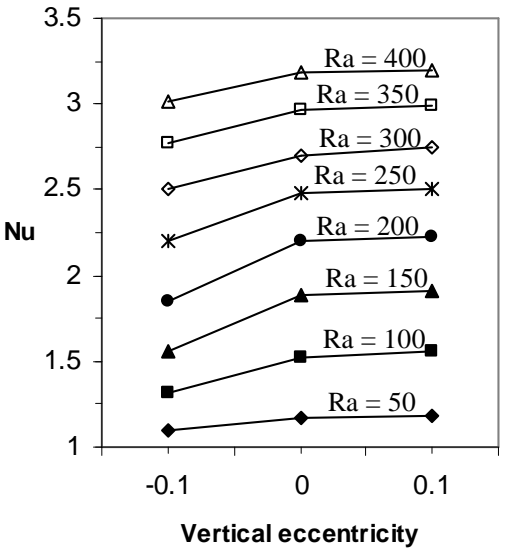

( b )

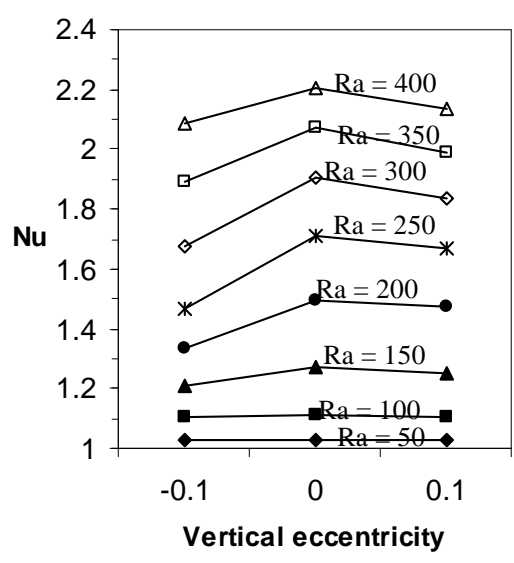

(c)

Fig ( 8 ) Nu versus $e_{y}$ for $e_{x}=0$ (a) $L_{b}=0.25$, (b) $L_{b}=0.3$, (c) $L_{b}=0.5$ 


\section{Conclusions}

The main purpose of this research is to find the influence of changing the horizontal and vertical position on heat transfer rate by natural convection.

1- The increasing in the Raleigh number resulting in increasing Nusselt number

2- Changing the horizontal position doesn't have a tangible effect on Nusselt number

3- The value of Nusselt number are highest when the body is closed to the upper surface of the cavity when the body size equal ( $0.25 \times 0.25)$ and $(0.3 \times 0.3)$.

4- The value of Nusselt number are highest when the body existed in the middle of the cavity, when the body size equal ( $0.5 \times 0.5)$.

5- The heat transfer rate from the upper surface of the body is highest than the other sides of body.

6- The increase of body size causes increase of heat transfer rate.

7- The horizontal position change gives rise to asymmetry stream function lines form.

\section{References}

1. Chan, B.K.C., Ivey, C.M., and Barry , J.M., "Natural Convection in Enclosed Porous Media with Rectangular Boundaries " J.Heat Transfer , 2,pp.21-27,1970.

2. Burns, P.J., Chow,L.C. and Tien, C.L. ,"Convection in vertical Slot Filled with Porous Insulation," Int.Heat Mass Transfer, 20,pp.919-926,1977.

3. Prasad, V., and Kulacki, F. A.," Natural Convection in a Vertical Porous Annulus", Int. J. Heat Mass Transfer, 27, pp. 207-219, 1984.

4. Oothuuizen, P.H.,"Mixed Convection Heat transfer from a Heated Horizontal Plate in aporous medium near an Impermeable surface ," J. Heat Transfer, 110, pp. 390-394, 1988.

5. Barbosa Mota, J. P., and Saatdjian, E.," Natural Convection between Eccentric Horizontal Porous Cylinders", Num.Meth. in Laminar and Turbulant Flow ,Vol.VII,Part 1,417-425,1996.

6. Al-Ani, O. B. H.," Natural Convective Heat Transfer from Rectangular Isothermal Body Embedded in Confined Porous Medium", M. Sc. Thesis, Mosul University. 1998.

7. Saeed, R. I" Numerical study of Natural Convective Heat Transfer from Horizontal cylinder Embedded square Porous Medium", M. Sc.Thesis, Mosul University. 2001

8. Eckert, E.R,G., and Darke, R.M., Analysis of Heat and Mass Transfer, McGraw-Hill, NewYork, pp.98-340, 1972.

9. Anderson, D. A., Tannehill, J. G., and Pietcher, R. H., " Computational Fluid Mechanics and Heat Transfer ", Mc Graw-Hill 1984.

10. Borse,G. J., FORTRAN77 and Numerical Method for Engineering, McGraw- Hill Book Company, Inc., 1985. 